\title{
CONVERGENCE OF THE NEWTON-TYPE METHOD FOR GENERALIZED EQUATIONS
}

\author{
M. H. Rashid ${ }^{1}$ and A. Sarder \\ Department of Mathematics, Faculty of Science, University of Rajshahi \\ Rajshahi-6205, Bangladesh \\ ${ }^{1}$ Corresponding author: harun_math@ru.ac.bd,
}

Received 04.09.2014

Accepted 08.09.2015

\begin{abstract}
Let $X$ and $Y$ be real or complex Banach spaces. Suppose that $f: X \rightarrow Y$ is a Frechet differentiable function and $F: X \rightrightarrows 2^{Y}$ is a set-valued mapping with closed graph. In the present paper, we study the Newton-type method for solving generalized equation $0 \in f(x)+F(x)$. We prove the existence of the sequence generated by the Newton-type method and establish local convergence of the sequence generated by this method for generalized equation.
\end{abstract}

Key words: Set-valued mapping, Generalized equation, Local convergence, Pseudo-Lipschitz mapping, Newton-type method

\section{Introduction}

The famous mathematician Robinson [13, 14] was introduced the concept of generalized equation, which was an extension of standard equations with no multi-valued part, as a general tool for describing, analyzing, and solving different problems in a unified manner. These kinds of generalized equation problems have been studied extensively; see for example [5, 13, 14]. Typical examples are systems of inequalities, variational inequalities, linear and non linear complementary problems, systems of nonlinear equations, equilibrium problems etc. Let $X$ and $Y$ be Banach spaces throughout this work unless otherwise stated. Let $f: X \rightarrow Y$ be a single-valued function and $F: X \rightrightarrows 2^{Y}$ be a setvalued mapping with closed graph. In this communication, we are concerned with the problem of approximating a point $x^{*}$ which satisfies the following generalized equation problem

$$
0 \in f\left(x^{*}\right)+F\left(x^{*}\right) \text {. }
$$

When the single-valued function involved in (1.1) is differentiable, Newton-like method can be considered to solve this generalized equation, such an approach has been used in many contributions to this subject [2, 3, 9]. To find an approximate solution of $(1,1)$ Dontchev [2] introduced the following classical Newton-type method:

$$
0 \in f\left(x_{k}\right)+\nabla f\left(x_{k}\right)\left(x_{k+1}-x_{k}\right)+F\left(x_{k+1}\right),
$$

where $\nabla f(x)$ denotes the Frechet derivative of $f$ at $x$. It is well known that if $F=\{0\}$, then (1.2) becomes the usual Newton method for solving the equation $f(x)=0$. If $F=\mathrm{R}_{+}^{m}$, the 
positive orthant in $\mathbb{R}^{m}$, then (1.1) describes a system of inequalities and (1.2) is a Newton-type method for solving such systems. Finally, the generalized equation (1.1) reduces to the classical variational inequalities:

$$
\text { find } x \in \Omega \text { with }\langle f(x), u-x\rangle \geq 0 \text { for all } u \in \Omega
$$

when $F(x)=N_{\Omega}(x)$ is the normal cone mapping generated by a convex and closed subset $\Omega \subseteq X$. Then the method (1.2) is known version of the Newton method for solving such problems.

Pietrus [9] showed that the sequence generated by the Newton-type method (1.2) converges superlinearly when $\nabla f$ is Holder continuous on a neighborhood of $\bar{x}$ and he also proved the stability of this method under mild conditions. Moreover, Geoffroy et al. [6] considered the following method as second degree Taylor polynomial expansion of $f$ for solving the smooth generalized equation (1.1):

$$
0 \in f\left(x_{k}\right)+\nabla f\left(x_{k}\right)\left(x_{k+1}-x_{k}\right)+\frac{1}{2} \nabla^{2} f\left(x_{k}\right)\left(x_{k+1}-x_{k}\right)^{2}+F\left(x_{k+1}\right),
$$

where $\nabla f(x)$ and $\nabla^{2} f(x)$ denote respectively the first and the second Frechet derivative of $f$ at $x$, and showed the existence of a sequence cubically converging to the solution of (1.1).

Our purpose in this study is to analyze local convergence of the Newton-type method (1.2) for solving generalized equation (1.1). The main tool is the Aubin continuous mapping for setvalued mapping, which was introduced by Aubin in the context of non smooth analysis, and studied by many mathematicians; see for examples [1, 8, 10, 15].

This paper is organized as follows: In Section 2, we recall some necessary notations, notions and preliminary results that will be used in the subsequent sections. In Section 3, we consider the Newton-type method (1.2) for solving the generalized equation (1.1), and establish existence and convergence of the sequence generated by the Newton-type method (1.2). In the last section, we give a summary of the major results of this study.

\section{Notations and Preliminaries}

Throughout, we assume that $X$ and $Y$ are real or complex Banach spaces. Let $x \in X$ and $r>0$. The closed ball centered at $x$ with radius $r$ denoted by $B(r, x)$.

The following definition of domain, inverse and graph of a function, distance from a point to a set, excess and pseudo-Lipschitz mapping are taken from $[4,11,12]$.

Definition 2.1. Let $F: X \rightrightarrows 2^{Y}$ be a set-valued mapping. Then the domain of $F$ is denoted by $\operatorname{dom} F$, and is defined by

$$
\operatorname{dom} F=\{x \in X: F(x) \neq \phi\} .
$$

The inverse of $F$, denoted by $F^{-1}$, is defined as

$$
F^{-1}(y)=\{x \in X: y \in F(x)\} .
$$


While the graph of $F$, denoted by gph $F$, is defined by

$$
\{(x, y) \in X \times Y: y \in F(x)\} .
$$

Definition 2.2. Let $X$ be a Banach space and $A$ be a subset of $X$. The distance from a point $x$ to a set $A$ is defined by

$$
\operatorname{dist}(x, A)=\inf \{\|x-y\|: y \in A\} .
$$

Definition 2.3. Let $X$ be a Banach space and $A, B \subseteq X$. The excess $e$ from the set $A$ to the set $B$ (also called the Hausdorff semidistance from $B$ to $A$ ) is given by

$$
e(B, A)=\sup \{\operatorname{dist}(x, A): x \in B\} .
$$

Definition 2.4. Let $F: X \rightrightarrows 2^{Y}$ be a set-valued mapping. Then $F$ is said to be pseudo-Lipschitz around $\left(x_{0}, y_{0}\right) \in \operatorname{gph} F$ with constant $M$ if there exist positive constants $\alpha, \beta>0$ such that

$$
e\left(F\left(x_{1}\right) \cap B\left(\beta, y_{0}\right), F\left(x_{2}\right)\right) \leq M\left\|x_{1}-x_{2}\right\|
$$

for every $x_{1}, x_{2} \in B\left(\alpha, x_{0}\right)$. When $F$ is single-valued, this corresponds to the usual concept of Lipschitz continuity.

The definition of Lipschitz continuity is equivalent to the definition of Aubin continuity which is given below:

A set-valued mapping $\Gamma: Y \rightrightarrows 2^{X}$ is said to be Aubin continuous at $\left(y_{0}, x_{0}\right) \in \operatorname{gph} \Gamma$ with constants $a, b$ and $M$ if for every $y_{1}, y_{2} \in B\left(b, y_{0}\right)$ and for every $x_{1} \in \Gamma\left(y_{1}\right) \cap B\left(a, x_{0}\right)$, there exists an $x_{2} \in \Gamma\left(y_{2}\right)$ with

$$
\left\|x_{1}-x_{2}\right\| \leq M\left\|y_{1}-y_{2}\right\| .
$$

The constant $M$ is called the modulus of Aubin continuity.

The definition of continuous map, Lipschitz continuous map and Holder continuous map are extracted from $[11,12]$.

Definition 2.5. Let $X$ and $Y$ be Banach spaces. Then the function $f: \Omega \subseteq X \rightarrow Y$ is said to be

(i) Continuous at $\bar{x} \in \Omega$ if for every $\varepsilon>0$, there exists a $\delta>0$ such that

$$
\|f(x)-f(\bar{x})\|<\varepsilon \text { for all points } x \in \Omega \text { for which }\|x-\bar{x}\|<\delta .
$$

(ii) Lipschitz continuous on an open subset of $X$ if there exists constant $c$ such that $\|f(x)-f(y)\| \leq c\|x-y\|$ for all $x$ and $y$ in the domain of $f$.

(iii) Holder continuous on an open subset of $X$ if there exists constant $c$ and $p \in(0,1]$ such that

$\|f(x)-f(y)\| \leq c\|x-y\|^{\mathrm{p}}$ for all $x$ and $y$ in the domain of $f$. The number $\mathrm{p}$ is called the exponent of the Holder condition. 
The definition of linear convergence, quadratic convergence and super linear convergence are taken from the book [7].

Definition 2.6. Let $\left\{x_{n}\right\}$ be a sequence which is converges to the number $\bar{x}$. Then the sequence $\left\{x_{n}\right\}$ is said to be

(i) Converges linearly to $\bar{x}$ if there exists a number $0<c<1$ such that

$$
\left\|x_{n+1}-\bar{x}\right\| \leq c\left\|x_{n}-\bar{x}\right\| .
$$

(ii) Converges quadratically to $\bar{x}$ if there exists a number $0<c<1$ such that

$$
\left\|x_{n+1}-\bar{x}\right\| \leq c\left\|x_{n}-\bar{x}\right\|^{2} .
$$

(iii) Converges superlinearly to $\bar{x}$ if there exists a number $0<c<1$ such that

$$
\left\|x_{n+1}-\bar{x}\right\| \leq c\left\|x_{n}-\bar{x}\right\|^{\mathrm{p}}, \quad(0<\mathrm{p} \leq 1) .
$$

We end this section with the following lemma, known as fixed point lemma, which can be found in [4].

Lemma 2.1. Let $X$ be a Banach space and $\Phi: \mathrm{X} \rightrightarrows 2^{\mathrm{X}}$ be a set-valued mapping. Let $\bar{x} \in X$; and let $r$ and $\lambda$ be such that, $0<\lambda<1$,

(a) $\operatorname{dist}(\bar{x}, \Phi(\bar{x}))<r(1-\lambda)$,

(b) $e\left(\Phi\left(x_{1}\right) \cap B(r, \bar{x}), \Phi\left(x_{2}\right)\right) \leq \lambda\left\|x_{1}-x_{2}\right\|$, for all $x_{1}, x_{2} \in B(r, \bar{x})$.

Then $\Phi$ has a fixed point in $B(r, \bar{x})$; that is, there exists $x \in B(r, \bar{x})$ such that $x \in \Phi(x)$. If $\Phi$ is single-valued, then $x$ is the unique fixed point of $\Phi$ in $B(r, \bar{x})$.

\section{Convergence Analysis}

This section is devoted to study the existence and the convergence of the sequence generated by the Newton-type method (1.2) for the generalized equation (1.1). Let $x \in X$ and define the mapping $Q_{x}$ by

$$
Q_{x}(\cdot)=f(x)+\nabla f(x)(\cdot-x)+F(\cdot) .
$$

Moreover, the following equivalence is clear for any $z \in X$ and $y \in Y$ :

$$
z \in Q_{x}^{-1}(y) \Leftrightarrow y \in f(x)+\nabla f(x)(z-x)+F(z) .
$$

In particular,

$$
x^{*} \in Q_{x^{*}}^{-1}\left(y^{*}\right) \text { for each }\left(x^{*}, y^{*}\right) \in \operatorname{gph}(f+F) .
$$

Let $\left(x^{*}, y^{*}\right) \in \operatorname{gph}(f+F)$ and let $\alpha>0, \beta>0$. Throughout this section, we assume that $B\left(\alpha, x^{*}\right) \subseteq \Omega \cap \operatorname{dom} F$, where $\Omega$ is the neighborhood of $x^{*}$.

The following lemma is useful and it plays a vital role in the convergence analysis of the Newtontype method (1.2) for solving the generalized equation (1.1). 
Lemma: 3.1. Let $X$ and $Y$ be Banach spaces and let $f: X \rightarrow Y$ be a function such that $\nabla f$ is Lipschitz continuous on an open subset of $X$ with Lipschitz constant $\kappa>0$. Then the following statements are equivalent:

(i) The mapping $(f+F)^{-1}$ is pseudo-Lipschitz around $\left(y^{*}, x^{*}\right)$.

(ii) The mapping $Q_{x^{*}}^{-1}(\cdot)$ is pseudo-Lipschitz around $\left(y^{*}, x^{*}\right)$.

Proof: Define a function $g: X \rightarrow Y$ by

$$
g(x)=-f(x)+f\left(x^{*}\right)+\nabla f\left(x^{*}\right)\left(x-x^{*}\right) .
$$

To complete the proof of this lemma, according to [4, Corollary 2], we need to show that $g$ is Lipschitz continuous at $x^{*}$. To do this, let $\varepsilon>0$, and take a positive number $\delta$ such that $\delta<\frac{\varepsilon}{2 \kappa}$. Then, for every $x_{1}, x_{2} \in B\left(\delta, x^{*}\right)$, we have

$$
\begin{aligned}
& \text { \| } g\left(x_{1}\right)-g\left(x_{2}\right) \| \\
& =\|\left(-f\left(x_{1}\right)+f\left(x^{*}\right)+\nabla f\left(x^{*}\right)\left(x_{1}-x^{*}\right)\right) \\
& -\left(-f\left(x_{2}\right)+f\left(x^{*}\right)+\nabla f\left(x^{*}\right)\left(x_{2}-x^{*}\right)\right) \| \\
& =\left\|f\left(x_{2}\right)-f\left(x_{1}\right)-\nabla f\left(x^{*}\right)\left(x_{2}-x_{1}\right)\right\| \\
& \leq \int_{0}^{1}\left\|\left(\nabla f\left(x_{1}+t\left(x_{2}-x_{1}\right)\right)-\nabla f\left(x_{1}\right)+\nabla f\left(x_{1}\right)-\nabla f\left(x^{*}\right)\right)\left(x_{2}-x_{1}\right)\right\| d t \\
& \leq \int_{0}^{1}\left\|\nabla f\left(x_{1}+t\left(x_{2}-x_{1}\right)\right)-\nabla f\left(x_{1}\right)\right\|\left\|x_{2}-x_{1}\right\| d t \\
& +\int_{0}^{1}\left\|\nabla f\left(x_{1}\right)-\nabla f\left(x^{*}\right)\right\|\left\|x_{2}-x_{1}\right\| d t \\
& \leq \kappa \int_{0}^{1}\left\|t\left(x_{2}-x_{1}\right)\right\| d t\left\|\left(x_{2}-x_{1}\right)\right\|+\kappa\left\|x_{1}-x^{*}\right\|\left\|x_{2}-x_{1}\right\| \\
& =\kappa\left[\frac{t^{2}}{2}\left\|x_{2}-x_{1}\right\|^{2}\right]_{0}^{1}+\kappa\left\|x_{1}-x^{*}\right\|\left\|x_{2}-x_{1}\right\| \\
& =\left(\frac{\kappa}{2}\left\|x_{2}-x_{1}\right\|+\kappa\left\|x_{1}-x^{*}\right\|\right)\left\|x_{2}-x_{1}\right\| \\
& \leq\left(\frac{\kappa}{2} \cdot 2 \delta+\kappa \delta\right)\left\|x_{2}-x_{1}\right\|=2 \kappa \delta\left\|x_{2}-x_{1}\right\| \\
& \leq 2 \kappa \cdot \frac{\varepsilon}{2 \kappa}\left\|x_{2}-x_{1}\right\|=\varepsilon\left\|x_{2}-x_{1}\right\| .
\end{aligned}
$$

Hence $g$ is Lipschitz at $x^{*}$ and this completes the proof of the lemma.

For our convenience, we define a function $Z_{x}: X \rightarrow Y$ by

$$
Z_{x}(\cdot)=f\left(x^{*}\right)+\nabla f\left(x^{*}\right)\left(\cdot-x^{*}\right)-f(x)-\nabla f(x)(\cdot-x)
$$


and a set-valued map $\Phi_{x}: X \rightrightarrows 2^{X}$ by

$$
\Phi_{x}(\cdot)=Q_{x^{*}}{ }^{-1}\left[Z_{x}(\cdot)\right]
$$

Then, for every $x^{\prime}, x^{\prime \prime} \in X$, we have

$$
\begin{aligned}
\left\|Z_{x}\left(x^{\prime}\right)-Z_{x}\left(x^{\prime \prime}\right)\right\|=\|( & \left.\nabla f\left(x^{*}\right)-\nabla f(x)\right)\left(x^{\prime}-x^{\prime \prime}\right) \| \\
& \leq\left\|\nabla f\left(x^{*}\right)-\nabla f(x)\right\|\left\|x^{\prime}-x^{\prime \prime}\right\|
\end{aligned}
$$

\subsection{Linear convergence}

If $\nabla f$ is continuous, then the following theorem gives us the linear convergence result.

Theorem 3.1 Let $x^{*}$ be a solution of (1.1). Suppose that $Q_{x^{*}}{ }^{-1}$ is pseudo-Lipschitz around $\left(0, x^{*}\right)$ with Lipschitz constant $\mathrm{M}$ and $\nabla f$ is continuous on a neighborhood $\Omega$ of $x^{*}$ with constant $L>0$. Then for every $c>2 M L$ one can find $\delta>0$ such that for every starting point $x_{0} \in B\left(\delta, x^{*}\right)$ there exists a sequence $\left\{x_{k}\right\}$ generated by (1.2), which satisfies

$$
\left\|x_{k+1}-x^{*}\right\| \leq c\left\|x_{k}-x^{*}\right\| .
$$

Proof: Fix $c>2 M L$ and since $Q_{x^{*}}{ }^{-1}$ is pseudo-Lipschitz with constant M, there exist positive constants $a$ and $b$ such that

$$
e\left(Q_{x^{*}}{ }^{-1}\left(y^{\prime}\right) \cap B\left(a, x^{*}\right), Q_{x^{*}}{ }^{-1}\left(y^{\prime \prime}\right)\right) \leq M\left\|y^{\prime}-y^{\prime \prime}\right\| \text {, for all } y^{\prime}, y^{\prime \prime} \in B(b, 0) .
$$

Choose $\delta>0$ and $B\left(\delta, x^{*}\right) \subset \Omega$ so that

$$
\delta \leq \min \left(\frac{a}{3}, 1, \frac{\mathrm{b}}{3 \mathrm{~L}}\right) \text { and } \quad M L \leq \frac{1}{2} .
$$

Define

$$
r_{x}=2 M L\left\|x-x^{*}\right\|, \quad \text { for all } x \in X .
$$

Let $x_{0} \in B\left(\delta, x^{*}\right), x_{0} \neq x^{*}$. Utilizing (3.7) in (3.8), we obtain that

$$
r_{x_{0}} \leq 2 M L \delta \leq 2 \cdot \frac{1}{2} \cdot \delta \leq \delta
$$

Now we will apply Lemma 2.1 to the map $\Phi_{x_{0}}$, defined in (3.3), with the following specifications:

$$
\bar{x}=x^{*}, \quad \lambda=\frac{1}{2} \text { and } r=r_{x_{0}} .
$$

Let us remark that $x^{*} \in Q_{x^{*}}{ }^{-1}(0) \cap B\left(\delta, x^{*}\right)$. From (3.6) and (3.7), we obtain for $x_{0} \in B\left(\delta, x^{*}\right)$, that

$$
\begin{aligned}
\operatorname{dist}\left(x^{*}, \Phi_{x_{0}}\left(x^{*}\right)\right) & \leq e\left(Q_{x^{*}}{ }^{-1}(0) B\left(\delta, x^{*}\right), \Phi_{x_{0}}\left(x^{*}\right)\right) \\
& \leq e\left(Q_{x^{*}}{ }^{-1}(0) \cap B\left(a, x^{*}\right), Q_{x^{*}}{ }^{-1}\left[Z_{x_{0}}\left(x^{*}\right)\right]\right) .
\end{aligned}
$$

Now, for all $x \in B\left(\delta, x^{*}\right)$, we have that

$$
\begin{aligned}
\| Z_{x_{0}}(x) & \|=\| f\left(x^{*}\right)+\nabla f\left(x^{*}\right)\left(x-x^{*}\right)-f\left(x_{0}\right)-\nabla f\left(x_{0}\right)\left(x-x_{0}\right) \| \\
& \leq\left\|f\left(x^{*}\right)-f\left(x_{0}\right)-\nabla f\left(x_{0}\right)\left(x^{*}-x_{0}\right)\right\|+\left\|\left(\nabla f\left(x^{*}\right)-\nabla f\left(x_{0}\right)\right)\left(x-x^{*}\right)\right\|
\end{aligned}
$$




$$
\begin{aligned}
& \leq \int_{0}^{1}\left\|\nabla f\left(x_{0}+t\left(x^{*}-x_{0}\right)-\nabla f\left(x_{0}\right)\right)\left(x^{*}-x_{0}\right)\right\| d t+\left\|\nabla f\left(x^{*}\right)-\nabla f\left(x_{0}\right)\right\|\left\|x-x^{*}\right\| \\
& \leq L \int_{0}^{1} d t\left\|x^{*}-x_{0}\right\|+L\left\|x-x^{*}\right\| \\
& =L\left\|x^{*}-x_{0}\right\|+L\left\|x-x^{*}\right\| \\
& \leq L \delta+L \delta=2 L \delta \leq 2 L \cdot \frac{b}{2 L}=b .
\end{aligned}
$$

This shows that $Z_{x_{0}}(x) \in B(b, 0)$ for all $x \in B\left(\delta, x^{*}\right)$. In particular, let $x=x^{*}$ in $(3.11)$, we get

$$
\begin{aligned}
& \left\|Z_{x_{0}}\left(x^{*}\right)\right\| \leq L\left\|x^{*}-x_{0}\right\| \\
& \leq L \delta \leq L \cdot \frac{b}{2 L} \leq b .
\end{aligned}
$$

Thus, we have that $Z_{x_{0}}\left(x^{*}\right) \in B(b, 0)$ for $x_{0} \in B\left(\delta, x^{*}\right)$.

Now, from the inequality (3.10), we have that

$$
\begin{aligned}
\operatorname{dist}\left(x^{*}, \Phi_{x_{0}}\left(x^{*}\right)\right) & \leq e\left(Q_{x^{*}}{ }^{-1}(0) \cap B\left(a, x^{*}\right), Q_{x^{*}}{ }^{-1}\left[Z_{x_{0}}\left(x^{*}\right)\right]\right) \\
& \leq\left\|Z_{x_{0}}\left(x^{*}\right)\right\| \\
& \leq M\left\|f\left(x^{*}\right)-f\left(x_{0}\right)-\nabla f\left(x_{0}\right)\left(x^{*}-x_{0}\right)\right\| \\
& =M \int_{0}^{1}\left\|\left(\nabla f\left(x_{0}+t\left(x^{*}-x_{0}\right)\right)-\nabla f\left(x_{0}\right)\right)\left(x^{*}-x_{0}\right)\right\| d t \\
& \leq M L \int_{0}^{1} d t\left\|x^{*}-x_{0}\right\|=M L\left\|x_{0}-x^{*}\right\| \\
& =\left(1-\frac{1}{2}\right) 2 M L\left\|x_{0}-x^{*}\right\|=(1-\lambda) r_{x_{0}} \\
& =(1-\lambda) r .
\end{aligned}
$$

That is, the condition (a) of Lemma 2.1 is hold.

Next, we show that condition (b) of Lemma 2.1 is satisfied. Further, using (3.4) and (3.7), for any $x^{\prime}, x^{\prime \prime} \in B\left(r_{x_{0}}, x^{*}\right)$ we obtain that

$$
\begin{aligned}
& e\left(\Phi_{x_{0}}\left(x^{\prime}\right) \cap B\left(r_{x_{0}}, x^{*}\right), \Phi_{x_{0}}\left(x^{\prime \prime}\right)\right) \\
& \leq e\left\{Q_{x^{*}}{ }^{-1}\left[Z_{x_{0}}\left(x^{\prime}\right)\right] \cap B\left(\delta, x^{*}\right), Q_{x^{*}}^{-1}\left[Z_{x_{0}}\left(x^{\prime \prime}\right)\right]\right\}
\end{aligned}
$$

$\leq M\left\|Z_{x_{0}}\left(x^{\prime}\right)-Z_{x_{0}}\left(x^{\prime \prime}\right)\right\| \leq M\left\|\nabla f\left(x^{*}\right)-\nabla f\left(x_{0}\right)\right\|\left\|x^{\prime}-x^{\prime \prime}\right\|$

$\leq M L\left\|x^{\prime}-x^{\prime \prime}\right\| \leq \frac{1}{2}\left\|x^{\prime}-x^{\prime \prime}\right\|=\lambda\left\|x^{\prime}-x^{\prime \prime}\right\|$.

This shows that the condition (b) of Lemma 2.1 is fulfilled. Hence, $\Phi_{x_{0}}$ has a fixed point $x_{1}$ in $B\left(r_{x_{0}}, x^{*}\right)$ such that

$$
\begin{aligned}
& x_{1} \in \Phi_{x_{0}}\left(x_{1}\right)=Q_{x^{*}}{ }^{-1}\left[Z_{x_{0}}\left(x_{1}\right)\right] \\
& \Rightarrow Z_{x_{0}}\left(x_{1}\right) \in Q_{x^{*}}\left(x_{1}\right) \\
& \Rightarrow 0 \in f\left(x_{0}\right)+\nabla f\left(x_{0}\right)\left(x_{1}-x_{0}\right)+F\left(x_{1}\right),
\end{aligned}
$$


which means that $x_{1}$ is obtained by the method (1.2) for initial point $x_{0}$. Thus $x_{1} \in B\left(r_{x_{0}}, x^{*}\right)$ implies that

$$
\left\|x_{1}-x^{*}\right\| \leq r_{x_{0}}=2 M L\left\|x_{0}-x^{*}\right\| \leq c\left\|x_{0}-x^{*}\right\| .
$$

Proceeding by induction, and applying the fixed point lemma 2.1 with $\bar{x}=x^{*}, \lambda=\frac{1}{2}, r=r_{x_{k}}$ to the map $\Phi_{x_{k}}$ in (3.3), we can deduce the existence of a fixed point $x_{k+1} \in B\left(\delta, x^{*}\right)$ such that

$$
\begin{aligned}
& x_{k+1} \in \Phi_{x_{k}}\left(x_{k+1}\right)=Q_{x^{*}}{ }^{-1}\left[Z_{x_{k}}\left(x_{k+1}\right)\right] \\
& \Rightarrow Z_{x_{k}}\left(x_{k+1}\right) \in Q_{x^{*}}\left(x_{k+1}\right) \\
& \Rightarrow 0 \in f\left(x_{k}\right)+\nabla f\left(x_{k}\right)\left(x_{k+1}-x_{k}\right)+F\left(x_{k+1}\right)
\end{aligned}
$$

and hence $\left\|x_{k+1}-x^{*}\right\| \leq c\left\|x_{k}-x^{*}\right\|$. This shows that (3.5) holds for $k$. This completes the proof of the theorem.

\subsection{Quadratic convergence}

In this subsection, we will show in the following theorem that if $\nabla f$ is Lipschitz continuous; the sequence generated by method (1.2) converges quadratically.

Theorem 3.2 Let $x^{*}$ be a solution of (1.1). Suppose that $Q_{x^{*}}{ }^{-1}$ is pseudo-Lipschitz around $\left(0, x^{*}\right)$ with Lipschitz constant $\mathrm{M}$ and $\nabla f$ is Lipschitz continuous on a neighborhood $\Omega$ of $x^{*}$ with constant $L>0$. Then for every $c^{\prime}>\frac{1}{2} M L$ one can find $\delta>0$ such that for every starting point $x_{0} \in B\left(\delta, x^{*}\right)$ there exists a sequence $\left\{x_{k}\right\}$ on a neighborhood $\Omega$ of $x^{*}$ generated by (1.2), which satisfies

$$
\left\|x_{k+1}-x^{*}\right\| \leq c^{\prime}\left\|x_{k}-x^{*}\right\|^{2} .
$$

Proof: Choose $c^{\prime}>0$ such that $c^{\prime}>\frac{1}{2} M L$. Since $Q_{x^{*}}{ }^{-1}$ is pseudo-Lipschitz with constant $M$, there exist positive constants $a$ and $b$ such that

$$
e\left(Q_{x^{*}}{ }^{-1}\left(y^{\prime}\right) \cap B\left(a, x^{*}\right), Q_{x^{*}}{ }^{-1}\left(y^{\prime \prime}\right)\right) \leq M\left\|y^{\prime}-y^{\prime \prime}\right\| \text {, for all } y^{\prime}, y^{\prime \prime} \in B(b, 0) .
$$

Let $\delta>0$ and $B\left(\delta, x^{*}\right) \subset \Omega$ so that

$$
\delta<\min \left\{\frac{a}{2}, \sqrt{\frac{2 b}{3 L}}, 1, \sqrt{\frac{1}{2 M L}}\right\} .
$$

Define

$$
r_{x}=M L\left\|x-x^{*}\right\|^{2}, \quad \text { for all } x \in X .
$$

Let $x_{0} \in B\left(\delta, x^{*}\right), x_{0} \neq x^{*}$. Using (3.14) in (3.15), we get

$$
r_{x_{0}}<M L \delta^{2}=M L \delta \cdot \delta \leq \frac{\delta}{2} \leq \delta .
$$

Now we will apply Lemma 2.1 to the map $\Phi_{x_{0}}$ in (3.3) with the following specifications

$$
\bar{x}=x^{*}, \quad \lambda=\frac{1}{2}, \quad r=r_{x_{0}} .
$$


Let us remark that $x^{*} \in Q_{x^{*}}{ }^{-1}(0) \cap B\left(\delta, x^{*}\right)$.

From (3.13) and (3.14), we obtain, for $x_{0} \in B\left(\delta, x^{*}\right)$, that

$$
\begin{aligned}
\operatorname{dist}\left(x^{*}, \Phi_{x_{0}}\left(x^{*}\right)\right) & \leq e\left(Q_{x^{*}}{ }^{-1}(0) \cap B\left(\delta, x^{*}\right), \Phi_{x_{0}}\left(x^{*}\right)\right) \\
& \leq e\left(Q_{x^{*}}{ }^{-1}(0) \cap B\left(a, x^{*}\right), Q_{x^{*}}{ }^{-1}\left[Z_{x_{0}}\left(x^{*}\right)\right]\right) .
\end{aligned}
$$

Now, for all $x \in B\left(\delta, x^{*}\right)$, we have that

$$
\begin{aligned}
& \left\|Z_{x_{0}}(x)\right\| \\
& =\left\|f\left(x^{*}\right)+\nabla f\left(x^{*}\right)\left(x-x^{*}\right)-f\left(x_{0}\right)-\nabla f\left(x_{0}\right)\left(x-x_{0}\right)\right\| \\
& \leq\left\|f\left(x^{*}\right)-f\left(x_{0}\right)-\nabla f\left(x_{0}\right)\left(x^{*}-x_{0}\right)\right\|+\left\|\left(\nabla f\left(x^{*}\right)-\nabla f\left(x_{0}\right)\right)\left(x-x^{*}\right)\right\| \\
& \leq \frac{L}{2}\left\|x^{*}-x_{0}\right\|^{2}+L\left\|x^{*}-x_{0}\right\|\left\|x-x^{*}\right\| \\
& \leq \frac{L}{2} \delta^{2}+L \delta^{2}=\frac{3}{2} L \delta^{2} \\
& \leq \frac{3 L}{2} \cdot \frac{2 b}{3 L}=b .
\end{aligned}
$$

So, we have $Z_{x_{0}}(x) \in B(b, 0)$ for all $x \in B\left(\delta, x^{*}\right)$. In particular, let $x=x^{*}$ in (3.18), we get

$$
\begin{aligned}
\left\|Z_{x_{0}}\left(x^{*}\right)\right\| & \leq \frac{L}{2}\left\|x^{*}-x_{0}\right\|^{2} \leq \frac{L}{2} \delta^{2} \\
& \leq \frac{L}{2} \cdot \frac{2 b}{3 L} \leq b
\end{aligned}
$$

Thus, we have $Z_{x_{0}}\left(x^{*}\right) \in B(b, 0)$.

Now, from the inequality (3.17), we get

$$
\begin{aligned}
\operatorname{dist}\left(x^{*}, \Phi_{x_{0}}\left(x^{*}\right)\right) \leq e & \left(Q_{x^{*}}{ }^{-1}(0) \cap B\left(a, x^{*}\right), Q_{x^{*}}{ }^{-1}\left[Z_{x_{0}}\left(x^{*}\right)\right]\right) \\
& \leq\left\|Z_{x_{0}}\left(x^{*}\right)\right\| \\
& \leq M\left\|f\left(x^{*}\right)-f\left(x_{0}\right)-\nabla f\left(x_{0}\right)\left(x^{*}-x_{0}\right)\right\| \\
& \leq M \int_{0}^{1}\left\|\left(\nabla f\left(x_{0}+t\left(x^{*}-x_{0}\right)\right)-\nabla f\left(x_{0}\right)\right)\left(x^{*}-x_{0}\right)\right\| d t \\
& \leq \frac{1}{2} M L\left\|x_{0}-x^{*}\right\|^{2} \\
& =\left(1-\frac{1}{2}\right) M L\left\|x_{0}-x^{*}\right\|^{2}=(1-\lambda) r_{x_{0}} \\
& =(1-\lambda) r .
\end{aligned}
$$

This means that condition (a) of Lemma 2.1 is hold.

Next, we show that condition (b) of Lemma 2.1 is also satisfied. To do this, let $x^{\prime}, x^{\prime \prime} \in B\left(r_{x_{0}}, x^{*}\right)$

. Then using (3.4) and (3.14), we obtain that 


$$
\begin{aligned}
& e\left(\Phi_{x_{0}}\left(x^{\prime}\right) \cap B\left(r_{x_{0}}, x^{*}\right), \Phi_{x_{0}}\left(x^{\prime \prime}\right)\right) \\
& \leq e\left\{Q_{x^{*}}{ }^{-1}\left[Z_{x_{0}}\left(x^{\prime}\right)\right] \cap B\left(\delta, x^{*}\right), Q_{x^{*}}{ }^{-1}\left[Z_{x_{0}}\left(x^{\prime \prime}\right)\right]\right\} \\
& \leq M\left\|Z_{x_{0}}\left(x^{\prime}\right)-Z_{x_{0}}\left(x^{\prime \prime}\right)\right\| \\
& \leq M\left\|\nabla f\left(x^{*}\right)-\nabla f\left(x_{0}\right)\right\|\left\|x^{\prime}-x^{\prime \prime}\right\| \\
& \leq M L\left\|x^{*}-x_{0}\right\|^{2}\left\|x^{\prime}-x^{\prime \prime}\right\| \\
& \leq M L \delta^{2}\left\|x^{\prime}-x^{\prime \prime}\right\| \leq \frac{1}{2}\left\|x^{\prime}-x^{\prime \prime}\right\| \\
& =\lambda\left\|x^{\prime}-x^{\prime \prime}\right\| ;
\end{aligned}
$$

that is, condition (b) of Lemma 2.1 is fulfilled. Hence, $\Phi_{x_{0}}$ has a fixed point $x_{1}$ in $B\left(r_{x_{0}}, x^{*}\right)$ such that

$$
\begin{aligned}
& x_{1} \in \Phi_{x_{0}}\left(x_{1}\right)=Q_{x^{*}}{ }^{-1}\left[Z_{x_{0}}\left(x_{1}\right)\right] \\
& \Rightarrow Z_{x_{0}}\left(x_{1}\right) \in Q_{x^{*}}\left(x_{1}\right) \\
& \Rightarrow 0 \in f\left(x_{0}\right)+\nabla f\left(x_{0}\right)\left(x_{1}-x_{0}\right)+F\left(x_{1}\right),
\end{aligned}
$$

which means that $x_{1}$ is obtained by the method (1.2) for initial point $x_{0}$. Thus $x_{1} \in B\left(r_{x_{0}}, x^{*}\right)$ implies that

$$
\left\|x_{1}-x^{*}\right\| \leq r_{x_{0}}=M L\left\|x_{0}-x^{*}\right\|^{2} \leq c^{\prime}\left\|x_{0}-x^{*}\right\|^{2}
$$

Proceeding by induction, and applying the fixed point lemma with $\bar{x}=x^{*}, \lambda=\frac{1}{2}, r=r_{x_{k}}$ to the map $\Phi_{x_{k}}$ in (3.3), we can deduce the existence of a fixed point $x_{k+1} \in B\left(\delta, x^{*}\right)$ such that

$$
\begin{aligned}
& x_{k+1} \in \Phi_{x_{k}}\left(x_{k+1}\right)=Q_{x^{*}}{ }^{-1}\left[Z_{x_{k}}\left(x_{k+1}\right)\right] \\
& \Rightarrow Z_{x_{k}}\left(x_{k+1}\right) \in Q_{x^{*}}\left(x_{k+1}\right) \\
& \Rightarrow 0 \in f\left(x_{k}\right)+\nabla f\left(x_{k}\right)\left(x_{k+1}-x_{k}\right)+F\left(x_{k+1}\right)
\end{aligned}
$$

and hence $\left\|x_{k+1}-x^{*}\right\| \leq c^{\prime}\left\|x_{k}-x^{*}\right\|^{2}$. This shows that (3.12) holds for $k$. This completes the proof of the theorem.

\subsection{Super linear convergence}

If $\nabla f$ is Holder continuous, the following theorem gives us the super linear convergence of the method (1.2).

Theorem 3.3 Let $x^{*}$ be a solution of (1.1). Let $p \in[0,1]$ and suppose that $Q_{x^{*}}{ }^{-1}$ is pseudoLipschitz around $\left(0, x^{*}\right)$ with Lipschitz constant $\mathrm{M}$ and $\nabla f$ is Holder continuous on a neighborhood $\Omega$ of $x^{*}$ with constant $L>0$. Then for every $\quad c^{\prime \prime}>\frac{M L}{p+1}$ one can find $\delta>0$ such that for every starting point $x_{0} \in B\left(\delta, x^{*}\right)$ there exists a sequence $\left\{x_{k}\right\}$ on a neighborhood $\Omega$ of $x^{*}$ generated by (1.2), which satisfies

$$
\left\|x_{k+1}-x^{*}\right\| \leq c^{\prime \prime}\left\|x_{k}-x^{*}\right\|^{p+1} .
$$


Proof: Fix $c^{\prime \prime}>\frac{M L}{p+1}$ and since $Q_{x^{*}}{ }^{-1}$ is pseudo-Lipschitz with constant $M$, there exist positive constants $a$ and $b$ such that

$$
e\left(Q_{x^{*}}{ }^{-1}\left(y^{\prime}\right) \cap B\left(a, x^{*}\right), Q_{x^{*}}{ }^{-1}\left(y^{\prime \prime}\right)\right) \leq M\left\|y^{\prime}-y^{\prime \prime}\right\| \text {, for all } y^{\prime}, y^{\prime \prime} \in B(b, 0) .
$$

Choose $\delta>0$ and $B\left(\delta, x^{*}\right) \subset \Omega$ so that

$$
\delta \leq \min \left\{\left(\frac{b(p+1)}{L(p+2)}\right)^{\frac{1}{p+1}},\left(\frac{p}{M L(p+1)}\right)^{\frac{1}{p}}\right\} .
$$

Define

$$
r_{x}=M L\left\|x-x^{*}\right\|^{p+1} .
$$

Let $x_{0} \in B\left(\delta, x^{*}\right), x_{0} \neq x^{*}$. Utilizing (3.21) in (3.22), we get

$$
r_{x_{0}} \leq M L \delta^{p+1}=M L \delta^{p} \cdot \delta \leq \frac{p}{p+1} \delta \leq \delta .
$$

Now we will apply Lemma 2.1 to the map $\Phi_{x_{0}}$ in (3.3) with the following specifications:

$$
\bar{x}=x^{*}, \quad \lambda=\frac{p}{p+1}, \quad r=r_{x_{0}} .
$$

Let us remark that $x^{*} \in Q_{x^{*}}{ }^{-1}(0) \cap B\left(\delta, x^{*}\right)$. From (3.20) and (3.21), we obtain, for $x_{0} \in$ $B\left(\delta, x^{*}\right)$, that

$$
\begin{aligned}
\operatorname{dist}\left(x^{*}, \Phi_{x_{0}}\left(x^{*}\right)\right) \leq e & \left(Q_{x^{*}}^{-1}(0) \cap B\left(\delta, x^{*}\right), \Phi_{x_{0}}\left(x^{*}\right)\right) \\
\leq & e\left(Q_{x^{*}}{ }^{-1}(0) \cap B\left(a, x^{*}\right), Q_{x^{*}}{ }^{-1}\left[Z_{x_{0}}\left(x^{*}\right)\right]\right) .
\end{aligned}
$$

Now, for all $x \in B\left(\delta, x^{*}\right)$, we have that

$$
\begin{aligned}
\| & z_{x_{0}}(x) \| \\
= & \left\|f\left(x^{*}\right)+\nabla f\left(x^{*}\right)\left(x-x^{*}\right)-f\left(x_{0}\right)-\nabla f\left(x_{0}\right)\left(x-x_{0}\right)\right\| \\
= & \left\|f\left(x^{*}\right)-f\left(x_{0}\right)-\nabla f\left(x_{0}\right)\left(x^{*}-x_{0}\right)\right\|+\left\|\left(\nabla f\left(x^{*}\right)-\nabla f\left(x_{0}\right)\right)\left(x-x^{*}\right)\right\| \\
\leq & \int_{0}^{1}\left\|\left(\nabla f\left(x_{0}+t\left(x^{*}-x_{0}\right)\right)-\nabla f\left(x_{0}\right)\right)\left(x^{*}-x_{0}\right)\right\| d t \\
& +\left\|\nabla f\left(x^{*}\right)-\nabla f\left(x_{0}\right)\right\|\left\|x-x^{*}\right\| \\
\leq & \int_{0}^{1}\left\|\nabla f\left(x_{0}+t\left(x^{*}-x_{0}\right)\right)-\nabla f\left(x_{0}\right)\right\| d t\left\|x^{*}-x_{0}\right\|+L\left\|x^{*}-x_{0}\right\|^{p}\left\|x-x^{*}\right\| \\
\leq & L \int_{0}^{1}\left\|t\left(x^{*}-x_{0}\right)\right\|^{p} d t\left\|x^{*}-x_{0}\right\|+L\left\|x^{*}-x_{0}\right\|^{p}\left\|x-x^{*}\right\| \\
= & \frac{L}{p+1}\left\|x^{*}-x_{0}\right\|^{p+1}+L\left\|x^{*}-x_{0}\right\|^{p}\left\|x-x^{*}\right\| \\
\leq & \frac{L}{p+1} \delta^{p+1}+L \delta^{p+1}=\frac{L(p+2)}{p+1} \delta^{p+1} .
\end{aligned}
$$


Then for $L(p+2) \delta^{p+1} \leq b(p+1)$ in (3.21), we have

$$
\left\|z_{x_{0}}(x)\right\| \leq b \text {. }
$$

So we have $Z_{x_{0}}(x) \in B(b, 0)$ for all $x \in B\left(\delta, x^{*}\right)$.

In particular, let $x=x^{*}$ in (3.25), we get

$$
\begin{aligned}
\left\|z_{x_{0}}\left(x^{*}\right)\right\| & \leq \frac{L}{p+1}\left\|x^{*}-x_{0}\right\|^{p+1} \\
& \leq \frac{L}{p+1} \delta^{p+1} \leq \frac{L}{p+1} \cdot \frac{b(p+1)}{L(p+2)} \leq b .
\end{aligned}
$$

Then by (3.21), we have that $Z_{x_{0}}\left(x^{*}\right) \in B(b, 0)$.

Now, from the inequality (3.24), we have that

$$
\begin{aligned}
\operatorname{dist}\left(x^{*}, \Phi_{x_{0}}\left(x^{*}\right)\right) & \leq e\left(Q_{x^{*}}{ }^{-1}(0) \cap B\left(a, x^{*}\right), Q_{x^{*}}{ }^{-1}\left[Z_{x_{0}}\left(x^{*}\right)\right]\right) \\
& \leq\left\|Z_{x_{0}}\left(x^{*}\right)\right\| \\
& \leq M\left\|f\left(x^{*}\right)-f\left(x_{0}\right)-\nabla f\left(x_{0}\right)\left(x^{*}-x_{0}\right)\right\| \\
& =M \int_{0}^{1}\left\|\left(\nabla f\left(x_{0}+t\left(x^{*}-x_{0}\right)\right)-\nabla f\left(x_{0}\right)\right)\left(x^{*}-x_{0}\right)\right\| d t \\
& \leq M \int_{0}^{1}\left\|\nabla f\left(x_{0}+t\left(x^{*}-x_{0}\right)\right)-\nabla f\left(x_{0}\right)\right\| d t\left\|x^{*}-x_{0}\right\| \\
& \leq M L \int_{0}^{1}\left\|t\left(x^{*}-x_{0}\right)\right\|^{p} d t\left\|x^{*}-x_{0}\right\| \\
& =M L\left[\frac{t^{p+1}}{p+1}\right]_{0}^{1}\left\|x^{*}-x_{0}\right\|^{p+1} \\
& =\frac{M L}{p+1}\left\|x^{*}-x_{0}\right\|^{p+1} \\
& =\left(1-\frac{p}{p+1}\right) M L\left\|x^{*}-x_{0}\right\|^{p+1}=(1-\lambda) r_{x_{0}} \\
& =(1-\lambda) r ;
\end{aligned}
$$

This shows that the condition (a) of Lemma 2.1 is satisfied.

Next, we show that condition (b) of Lemma 2.1 also holds. To complete this, let $x^{\prime}, x^{\prime \prime} \in$ $B\left(r_{x_{0}}, x^{*}\right)$. Now, using (3.4) and (3.21), we obtain that

$$
\begin{aligned}
& e\left(\Phi_{x_{0}}\left(x^{\prime}\right) \cap B\left(r_{x_{0}}, x^{*}\right), \Phi_{x_{0}}\left(x^{\prime \prime}\right)\right) \\
& \leq e\left\{Q_{x^{*}}{ }^{-1}\left[Z_{x_{0}}\left(x^{\prime}\right)\right] \cap B\left(\delta, x^{*}\right), Q_{x^{*}}{ }^{-1}\left[Z_{x_{0}}\left(x^{\prime \prime}\right)\right]\right\} \\
\leq & M\left\|Z_{x_{0}}\left(x^{\prime}\right)-Z_{x_{0}}\left(x^{\prime \prime}\right)\right\| \leq M\left\|\nabla f\left(x^{*}\right)-\nabla f\left(x_{0}\right)\right\|\left\|x^{\prime}-x^{\prime \prime}\right\| \\
\leq & M L\left\|x^{*}-x_{0}\right\|^{p}\left\|x^{\prime}-x^{\prime \prime}\right\| \leq M L \delta^{p}\left\|x^{\prime}-x^{\prime \prime}\right\|
\end{aligned}
$$




$$
\leq \frac{p}{p+1}\left\|x^{\prime}-x^{\prime \prime}\right\|=\lambda\left\|x^{\prime}-x^{\prime \prime}\right\| ;
$$

This indicates that the condition (b) of Lemma 2.1 is fulfilled. Hence, $\Phi_{x_{0}}$ has a fixed point $x_{1}$ in $B\left(r_{x_{0}}, x^{*}\right)$ such that

$$
\begin{aligned}
& x_{1} \in \Phi_{x_{0}}\left(x_{1}\right)=Q_{x^{*}}{ }^{-1}\left[Z_{x_{0}}\left(x_{1}\right)\right] \\
& \Rightarrow Z_{x_{0}}\left(x_{1}\right) \in Q_{x^{*}}\left(x_{1}\right) \\
& \Rightarrow 0 \in f\left(x_{0}\right)+\nabla f\left(x_{0}\right)\left(x_{1}-x_{0}\right)+F\left(x_{1}\right),
\end{aligned}
$$

which means that $x_{1}$ is obtained by the method (1.2) for initial point $x_{0}$. Thus $x_{1} \in B\left(r_{x_{0}}, x^{*}\right)$ implies that

$$
\left\|x_{1}-x^{*}\right\| \leq r_{x_{0}}=M L\left\|x_{0}-x^{*}\right\|^{p+1} \leq c^{\prime \prime}\left\|x_{0}-x^{*}\right\|^{p+1} .
$$

Proceeding by induction, and applying the fixed point lemma with $\bar{x}=x^{*}, \lambda=\frac{p}{p+1}, r=r_{x_{k}}$ to the map $\Phi_{x_{k}}$ in (3.3), we can deduce the existence of a fixed point $x_{k+1} \in B\left(\delta, x^{*}\right)$ such that

$$
\begin{aligned}
& x_{k+1} \in \Phi_{x_{k}}\left(x_{k+1}\right)=Q_{x^{*}}{ }^{-1}\left[Z_{x_{k}}\left(x_{k+1}\right)\right] \\
& \Rightarrow Z_{x_{k}}\left(x_{k+1}\right) \in Q_{x^{*}}\left(x_{k+1}\right) \\
& \Rightarrow 0 \in f\left(x_{k}\right)+\nabla f\left(x_{k}\right)\left(x_{k+1}-x_{k}\right)+F\left(x_{k+1}\right)
\end{aligned}
$$

and hence

$$
\left\|x_{k+1}-x^{*}\right\| \leq c^{\prime \prime}\left\|x_{k}-x^{*}\right\|^{p+1}
$$

This shows that (3.19) holds for $k$. This completes the proof of the theorem.

\section{Concluding Remarks}

We have established the local convergence of the Newton-type method under the assumptions that $Q_{x^{*}}{ }^{-1}(\cdot)$ is pseudo-Lipschitz and $\nabla f$ is continuous, Lipschitz-continuous and Holder continuous. We have shown that if $\nabla f$ is continuous, Lipschitz continuous and Holder continuous then the Newton-type method is linearly convergent, quadratically convergent and super linearly convergent respectively. This investigation improves and extends the results corresponding to [2].

\section{REFERENCES}

[1] Aubin J. P. (1984), Lipschitz behavior of solutions to convex minimization problems, Math. Oper. Res. $9: 87-111$

[2] Dontchev A. L. (1996.), Local convergence of the Newton method for generalized equations, R. Acad. Sc. Paris Ser I Math., 322 : 327-331.

[3] Dontchev A. L. (1996), Local analysis of a Newton-type method based on partial linearization, Lectures in Applied Mathematics, $32: 295-306$.

[4] Dontchev A. L. and Hager W. W. (1994), An inverse function theorem for set-valued maps, Proc. Amer. Math. Soc., 121: 481-489.

[5] Ferris M. C. and Pang J. S. (1997), Engineering and economic applications of complementary problems, SIAM Rev., 39 : 669-713. 
[6] Geoffroy M. H., Hilout S. and Pietrus A. (2003), Acceleration of convenience in Dontchev's iterative methods for solving variational inclusions, Serdica Math. J., 45-54.

[7] Kendell Atkinson A. (1988), An introduction to numerical analysis ( $2^{\text {nd }}$ edition), John Willey and Sons.

[8] Mordukhovich B. S. (1992), Sensitivity analysis in nonsmooth optimization: Theoretical Aspects of Industrial Design (D. A. Field and V. Komkov, eds.). SIAM Proc. Appl. Math. 58 : 32-46.

[9] Pietrus A. (2000), Does Newtons method for set -valued maps converges uniformly in mild differentiability context? Rev. Colomb. Mat., 34 : 49-56.

[10] Qi L. (1993), Convergence analysis of some algorithms for solving non-smooth equations, Math. Oper. Res., $18: 227-244$.

[11] Rashid M. H., Yu S. H., Li C. and Wu S. Y. (2013), Convergence analysis of Gauss-Newton method for Lipschitz-like mappings, J. Optim Theory Appl., 158(1) : 216-233.

[12] Rashid M. H., Wang J. H. and Li C. (2012), Convergence analysis of a method for variational inclusions, Applicable Analysis, 91(10) : 1943-1956.

[13] Robinson S. M. (1979), Generalized equations and their solutions, part I: basic theory. Math. Program. Stud., 10 : 128-141.

[14] Robinson S. M. (1982), Generalized equations and their solutions, part II: applications to nonlinear programming, Math. Program. Stud., 19 : 200-221.

[15] Rockfellar R. T. (1984), Lipschitzian properties of multifunctions, Nonlin. Anal., 9 : 867-885. 